\title{
Philosophiques
}

\section{Une analyse de la notion d'objectivité}

\section{Yvon Provençal}

Volume 14, numéro 2, automne 1987

URI : https://id.erudit.org/iderudit/027017ar

DOI : https://doi.org/10.7202/027017ar

Aller au sommaire du numéro

Éditeur(s)

Société de philosophie du Québec

ISSN

0316-2923 (imprimé)

1492-1391 (numérique)

Découvrir la revue

Citer cet article

Provençal, Y. (1987). Une analyse de la notion d'objectivité. Philosophiques, 14(2), 361-380. https://doi.org/10.7202/027017ar
Résumé de l'article

La notion d'objectivité fait l'objet d'une étude qui s'inscrit dans une visée globale, ce qui doit s'entendre dans le sens d'une globalité des savoirs rationnels, auxquels on tente ici d'assigner des ordres d'objectivité, et dans le sens d'une globalité historique, où il est envisagé de faire correspondre ces ordres d'objectivité à quelques grandes époques de l'histoire de la pensée rationnelle depuis l'Antiquité. On se penchera notamment sur la signification profonde des rapports intersubjectifs dans les constitutions objectives. Une clarification de la signification de l'induction dans les sciences expérimentales pourra en résulter.
Ce document est protégé par la loi sur le droit d'auteur. L'utilisation des services d'Érudit (y compris la reproduction) est assujettie à sa politique d'utilisation que vous pouvez consulter en ligne.

https://apropos.erudit.org/fr/usagers/politique-dutilisation/ 


\title{
UNE ANALYSE DE LA NOTION D'OBJECTIVITÉ
}

\author{
par Yvon Provençal
}

\begin{abstract}
RÉSUMÉ. La notion d'objectivité fait l'objet d'une étude qui s'inscrit dans une visée globale, ce qui doit s'entendre dans le sens d'une globalité des savoirs rationnels, auxquels on tente ici d'assigner des ordres d'objectivité, et dans le sens d'une globalité historique, où il est envisagé de faire correspondre ces ordres d'objectivité à quelques grandes époques de l'histoire de la pensée rationnelle depuis l'Antiquité. On se penchera notamment sur la signification profonde des rapports intersubjectifs dans les constitutions objectives. Une clarification de la signification de l'induction dans les sciences expérimentales pourra en résulter.
\end{abstract}

ABSTRACT. We examine the notion of objectivity in a global aim, i.e. along the line of the main types of rational knowledges and along the line of the history since the Antiquity. We develop the idea of many orders of objectivity each of which corresponding to types of knowledge and to great periods in the history of rational thinking. In particular we attempt to consider the deep significance of intersubjectivity in relation to objectivity. As a result the significance of the induction in the experimental sciences should become clearer.

Il est proposé ici de faire une étude de la notion d'objectivité aussi bien dans le rôle qu'elle joue au sein du discours scientifique — rôle d'ailleurs inhérent au statut de la science même - que dans l'utilisation générale qu'on en fait, où cette notion peut être associée au caractère indépendant des jugements et à leur qualité d'universalité. L'approche de cet article se démarque des approches connues en ce que je tenterai de prendre un point de vue surélevé ou plus global sur l'ensemble de la pensée rationnelle (expression souvent associée à celle de la pensée « occidentale»). Il faudra pour cela établir une sorte de métalangage épistémologique, où certaines notions seront définies qui nous amèneront à reconnaître des 
types, ou plus précisément des ordres d'objectivité. Ceux-ci seront définis à partir de la mise en évidence de certains présupposés, dénotés ici par l'expression de "référence implicite», dont la structure montrera clairement des dénivellations en rapport avec des domaines du savoir (e.g. ceux qui sont liés au raisonnement mathématique, à la vérification expérimentale) et avec des époques de l'histoire de la pensée.

Une définition de $A$. Lalande pourra d'abord nous servir de point de repère pour envisager la notion d'objectivité dans son sens le plus généralement admis. Cet auteur peut être cité utilement à cette fin même si l'intention est ici de dépasser ses définitions. Des auteurs comme Kant, Russel, Bachelard ou Popper notamment nous fourniront ensuite certains éléments essentiels de caractérisation pour situer la notion d'objectivité au sens moderne. Un bref historique sera fait où l'on pourra constater comment la généalogie de la notion moderne d'objectivité remonte jusqu'à l'Antiquité grecque présocratique et reproduit généralement les ordres d'objectivité tels que définis ici. Nous verrons ensuite comment ces notions peuvent jeter une lumière nouvelle sur le problème épistémologique de l'induction dans les sciences expérimentales. Essentiellement celle-ci correspond à l'actualisation d'un des ordres de l'objectivité, soit celui qui suppose une référence intersubjective, où plusieurs sujets individuels peuvent communiquer leurs expériences entre eux. Il sera enfin intéressant d'arguer que plusieurs ordres d'objectivité existent, même au-delà de ceux qui correspondent aux constitutions épistémologiques habituelles, et qu'une certaine libération conceptuelle peut en résulter.

\section{Les ordres d'objectivité}

Notons d'abord que ce qu'on appelle « réalité subjective » ou «impression subjective» peut être caractérisé, selon un mode descriptif dont l'intention apparaîtra clairement, comme étant ce qui appartient à un sujet individuel et qui diffère, ou varie, d'un sujet à l'autre. Le « subjectif » est, en ce sens, d'une part constant ou invariant (au moins pendant une certaine durée) en un sujet donné, d'autre part inconstant ou variable sur un groupe comprenant plusieurs sujets. Par exemple la préférence d'un individu pour un certains mets peut très bien persister en lui tout en n'étant 
nullement partagée par d'autres individus. Cette préférence est subjective et d'ailleurs bien réelle pour l'individu en cause. Sa réalité apparaîtra cependant relative aux yeux des autres.

Généralisons quelque peu a partir de ce point de départ. Nous pouvons admettre que l'objet réel possède généralement une certaine invariance sur un domaine de variation. Celui-ci peut consister par exemple en un ensemble d'individus dans un groupe, ou bien encore en un ensemble de moments individuels dans un seul individu. Considérons le premier de ces deux cas, qui peut correspondre à ce qu'on appelle couramment objectivité dans le domaine des sciences expérimentales. L'inhérence de l'objectivité à l'intersubjectivité, c'est-à-dire leur étroite relation, a été constatée déjà par plusieurs épistémologues ou penseurs modernes (nous y reviendrons ci-dessous). Dans ce cas, la chose objective doit être la même, ou s'avérer invariante, pour les différents individus dans une communauté de sujets identifiables entre autres aux chercheurs eux-mêmes de la science considérée. Nous pouvons voir que la caractérisation de la chose réelle en termes d'une invariance sur un domaine de variation met en lumière un lien entre le «subjectif » et l'«objectif», tout en révélant ce qui fait l'essentiel de leur opposition. Ce lien et cette opposition seront mieux exprimés si nous introduisons la notion générale de base de référence comme suit. La base de référence est l'ordre de l'ensemble d'instances subjectives qui constitue le domaine de variation, où l'instance subjective représente le moment individuel d'un sujet. L'expression d'«instance subjective» prend cependant un sens plus particulier dans le contexte de notre approche. Son utilité réside dans sa capacité de généralisation selon divers ordres tels que décrits cidessous. Ainsi l'individu sera défini comme un ensemble d'instances subjectives; la communauté (ou la «société», ou encore le " groupe ») sera définie comme un ensemble d'individus, et donc comme un ensemble d'ensembles d'instances subjectives. Par conséquent l'individu est un ensemble d'ordre un d'instances subjectives et la communauté est un ensemble d'ordre deux d'instances subjectives. Par extension l'objectivité-1 sera celle de la chose invariante sur un ensemble d'ordre un d'instances subjectives, soit un sujet individuel, et l'objectivité-2 sera celle de la chose invariante sur un ensemble d'ordre deux d'instances subjectives, soit une communauté de sujets individuels. L'objectivité- 2 correspond 
ainsi à l'objectivité dans le sens courant (notamment pour les sciences expérimentales) et l'objectivité-1 correspond à ce qui est appelé plutôt subjectivité ${ }^{1}$. Les opinions, les goûts et les expériences personnelles relèvent de l'ordre un d'objectivité puisque la réalité alors considérée varie d'individu à individu tout en étant, en chacun, claire et reconnue par l'individu lui-même au travers de ses moments successifs, tels qu'englobés dans sa mémoire. Par extension, ou par généralisation du procédé, l'objectivité-0 représente le cas limite de l'impression fugitive et de l'illusion. Celles-ci, contrairement à la réalité subjective de l'expérience individuelle, ne sont pas reconnues par l'individu comme des réalités associées à sa personne. Même seul, il peut les rejeter comme dénuées de substance. D'autre part, l'objectivité-3 représente une autre espèce d'objectivité, ou de vérité, dont l'ordre est supérieur à celui de l'objectivité dans le sens courant. Si cette dernière suppose l'ordre deux comme base de référence, disons une référence intersubjective, ou sociale (où la « société » de référence est définie simplement comme un ensemble de sujets individuels en rapport éventuel les uns avec les autres), l'objectivité- 3 suppose une référence à un ensemble de communautés distinctes. Dans ce cas, l'histoire au sens moderne, dont les différents moments s'associent éventuellement à des sociétés humaines qui changent, ou encore la communauté internationale, dont les éléments sont des peuples ou des nations, peuvent représenter la base de référence. La possibilité conceptuelle de varier sur un ensemble de sociétés distinctes peut donner lieu à un type de réalité objective nécessitant, pour être comprise, des considérations différentielles sur plusieurs sociétés, ou encore sur plusieurs moments historiques ${ }^{2}$.

Une séquence ordonnée peut donc être dégagée :

Objectivité-0 : l'impression fugitive, l'illusion.

Objectivité-1: le subjectif individuel, l'opinion.

Objectivité-2: l'objectif au sens courant, le scientifique.

Objectivité-3: la vérité historique au sens moderne.

1. C'est le passage de l'intrasubjectif à l'intersubjectif pour la base de référence, qui peut être également décrit en termes du passage de la dimension individuelle, ensemble linéaire des moments temporels de l'individu, à la dimension sociale, qui est double puisqu'elle comporte la dimension individuelle répétée indéfiniment.

2. Nous verrons plus loin comment Lucien Goldmann considère une objectivité propre aux sciences humaines qui fonctionnerait sur la considération essentielle de groupes sociaux distincts. 
Nous verrons que les caractérisations de l'objectivité proprement dite rejoignent bien ce que nous avons identifié comme l'objectivité-2, avec une base de référence intersubjective.

\section{Définitions existantes de l'objectivité}

Les définitions, ou les caractérisations, existantes de l'objectivité se contentent souvent de peu. Elles tendent généralement à suggérer la nature et l'importance de la notion d'objectivité sans pousser l'interrogation au-delà de certaines limites. Je me servirai de la définition qu'en donne $A$. Lalande pour illustrer mon propos. Cet auteur propose d'employer le mot "objectif» au sens de l'opposition au subjectif comme s'oppose l'universel à l'individuel: "Cette opposition, écrit-il, est précise, centrale, conforme à l'usage des historiens et des savants... elle contient virtuellement tout ce qu'il y a de solide dans les autres distinctions auxquelles ces mots ont été appliqués. " ${ }^{3}$ L'« universel » ici opposé à l'individuel s'entend évidemment au sens d'un univers d'individus (c'est-à-dire sans accorder de pertinence à l'existence possible d'une ou plusieurs sociétés distinctes), et donc d'un ensemble d'ordre deux d'instances subjectives, ce qui rejoint alors le sens donné à l'objectivité-2 cidessus. Lalande n'a pas tort, à mon avis, de dire que cette opposition de l'universel et de l'individuel contient «tout ce qu'il y a de solide» dans les distinctions auxquelles les mots «objectif » et «subjectif» ont été appliqués. Examinons quelques-unes de ces distinctions.

On peut d'abord caractériser l'objectivité avec des termes qui expriment la constance de ce qui subsiste, de ce qui se répète, de ce qui est reconnaissable par tous. On parle alors d'invariance ou de fidélité au réel. En général, on se contente par là de suggérer une stabilité qui s'oppose à la mouvance de l'informe ou de l'irréel sans spécifier exactement par rapport à quoi il y a invariance ou fidélité. Il y aurait un fond stable sur quoi se détacheraient du mouvement et du devenir. Pour Kant, la substance est un concept a priori, dont le principe (parmi les principes dits des « analogies de l'expérience ») permet de penser le variable : seule la permanence de la substance

3. A. LALANDE, Vocabulaire technique et critique de la philosophie, Paris, P. U. F.; première éd., 1926; quatorzième éd., 1983, p. 701. 
permet aux variations d'exister ${ }^{4}$. Si toutefois on refuse l'approche aprioriste, on peut chercher à comprendre la réalité (comme celle de la «substance ») comme étant elle-même une chose considérée stable sur un fond de référence par rapport auquel elle s'en dégagera comme invariante. Cela n'équivaut d'ailleurs pas à l'interprétation héraclitéenne, selon laquelle le stable ne serait que l'apparence accidentelle de la matière constamment en devenir, parce que ce devenir lui-même peut être pensé réel à partir d'une référence implicite de base sur laquelle des variations d'instances subjectives sont effectuées.

Dans la modernité on tend à se passer du concept de substance. On lui préfère par exemple les notions d'objet ou de fait objectif (qui, elles, ne prendront tout leur sens que selon la référence historique ou universelle au sens moderne). On peut trouver cette attitude chez plusieurs auteurs modernes. Considérons en particulier B. Russell et sa conception de la persistance des objets sensibles. Pour lui, la croyance à la persistance des objets sensibles en général, lorsque nous ne les percevons pas, est une donnée peu solide (par opposition aux données solides de la perception directe), au même titre d'ailleurs que la croyance à l'existence d'autres esprits ${ }^{5}$. Ce qui conduit à cette croyance est selon cet auteur un processus analogue à celui de l'acquisition du langage. Dans ce cas, un «bruit verbal» est associé à quelque élément notable de l'environnement qui est perçu ${ }^{6}$. Toutefois le mot luimême doit être considéré comme un universel, entendu dans le sens d'une classe d'illustrations particulières. Le rôle que prend le langage peut signifier par la suite une implication de l'intersubjectivité dans la mesure où l'on admet avec Russell qu'il y a un degré de vérification possible de la persistance des choses au moyen des observations d'un homme isolé, mais que cela « ne nous mène pas loin dans l'établissement d'une science complète ${ }^{7}$. »

4. E. Kant, Critique de la raison pure, "Analytique des principes »; trad. J. Barni, Paris, Flammarion, 1976, p. 223 sq.

5. B. Russell, Méthode scientifique en philosophie, trad. Ph. Devaux, Paris, Vrin, 1929, p. $60-69$.

6. B. Russel., Signification et Vérité, trad. Ph. Devaux, Paris, Flammation, 1959, p. 26-29.

7. Id., p. 40 
Cette question de la persistance des objets sensibles peut utilement être interprétée de la façon suivante. Examinons ce qui doit varier pour qu'une stabilité d'autre chose en ressorte. Nous avons l'allusion à cette base implicite dans une définition de l'objectivité telle que celle qu'a donnée A. Lalande. Quand il est dit que l'objectif s'oppose au subjectif comme l'universel s'oppose à l'individuel, nous pouvons saisir ce par rapport à quoi il doit y avoir invariance ou fidélité de la chose dite objective. Une réalité a une signification universelle par opposition à individuelle si elle reste invariante sur un univers d'individus, c'est-à-dire sur une base intersubjective. La fidélité au réel est celle qui s'attache à un univers intersubjectif. En ce sens, il est par suite possible d'interchanger les sujets témoins de la chose sans modifier la chose, et c'est ce qui fait qu'il y a fidélité descriptive et que la chose est objective.

Cependant des précisions supplémentaires sont requises sur ce qui fait l'aptitude ou la compétence des témoins. Lalande distingue en droit l'objectif du subjectif ${ }^{8}$. Cela signifie que tout individu peut en principe être témoin de la réalité dite objective sans que cela n'équivaille à une exigence de fait qui serait irréalisable. Nous devrions d'ailleurs la juger irréalisable non parce qu'il serait trop long, ou trop difficile, d'impliquer tous les individus existants, mais plutôt parce que ces individus existant en fait ont déjà par le fait même une réalité objective et qu'il y aurait un cercle vicieux à justifier l'objectif par l'objectif. C'est ce que signifie ce droit de reconnaître l'objectif, qui est préalable à la constitution de tout fait objectif 9 .

Une formulation de Kant apporte une précision supplémentaire ${ }^{10}$ : "Quand cet acte [tenir quelque chose pour vrai] est valable pour chacun, pour quiconque du moins a la raison, le principe en est objectivement suffisant, ... » Cela suggère bien de restreindre le groupe de référence à ceux qui ont « la raison ». C'est peut-être une autre façon d'exprimer le droit mentionné ci-dessus comme condition. La légitimité appartient à ceux qui ont la raison.

\footnotetext{
8. A. Lalande, ibidem.

9. Ces remarques rejoignent quelque peu les critiques du psychologisme qu'ont formulées des auteurs comme E. Husseri ou K. R. POPPER par exemple.

10. E. KANT, op. cit., "Méthodologie transcendantale», p. 611.
} 
"Être vérifiable en droit par quiconque» signifie ainsi : «être vérifiable en droit par quiconque a la raison. " L'individu de référence, s'il a la raison, peut reconnaître le groupe en possession du droit.

On peut aller plus loin dans les restrictions. K. R. Popper exige pour l'objectivité des énoncés scientifiques qu'ils puissent " être intersubjectivement soumis à des tests » ou, plus généralement, qu'ils puissent être soumis à la critique intersubjective ou au contrôle mutuel par examen critique ${ }^{11}$. D'une façon plus tranchante, G. Bachelard affirme que «le consensus n'est pas la base du rationnel parce que le consensus de l'expérience vulgaire est en contradiction avec celui de l'expérience savante ${ }^{12}$. Avec ce type d'exigence, de Popper ou de Bachelard, la condition de droit devient plus restrictive et même, pourrions-nous dire, change de nature. Pour juger qu'un individu, ou un groupe, est en droit un témoin dans l'intersubjectivité pour constituer, ou reconnaître, la réalité objective, il lui faut une qualification institutionnalisée qui constitue le critère permettant de reconnaître la société savante. Il serait possible de montrer que l'ordre trois comme base de référence, c'est-à-dire l'ordre historique, est alors requis. En effet l'homme de science, apte à juger du groupe de référence ou à en faire partie, sera qualifié à partir d'institutions sociales ellesmêmes justifiées par leur place et leur rôle dans le développement historique et moderne de la science ou du savoir en général. Notons ici que le caractère particulier de l'institution sociale importe peu et que c'est plutôt le principe de sa nécessité qui est en cause de même que son inscription dans une histoire.

\section{Généalogie de la notion d'objectivité}

Nous avons vu que l'objectivité au sens courant se définit dans une opposition au subjectif. Toute la pensée occidentale, depuis les Présocratiques, semble fonctionner selon des rapports d'opposition qui tiennent à un motif semblable, suivant les époques. Que nous considérions l'opposition présocratique de la vérité au

11. K.R. POPPER, La logique de la découverte scientifique, trad. N. Thyssen-Rutten et P. Devaux, Paris, Payot, 1973, p. 41.

12. G. BaCHELARD, Le rationalisme appliqué, Paris, P.U.F., 1949, p. 22. 
mythe, l'opposition platonicienne de la science à l'opinion, l'opposition moderne ${ }^{13}$ du sens historique aux modes et aux validations momentanées, il s'agit chaque fois d'une opposition fondamentale pour l'époque où elle apparaît parce qu'elle fournit essentiellement une base de constitution épistémologique. Il est possible de montrer, à mon avis, que ces oppositions fondamentales ont une structure analogue en termes de référence implicite et qu'elles diffèrent seulement par l'ordre de cette référence.

Au début de la pensée occidentale, Parménide commande, à partir de sa vision unique et épurée de l'être, de rejeter la contradiction logique à l'intérieur de son propre discours. Plus tard, Platon pose l'exigence d'universalité pour le savoir vrai, ce qui signifie dans ce cas que la vérité doit être identique pour tous les sujets individuels dans une communauté dialoguante. Enfin, dans la modernité, l'exigence correspondante se retrouve dans l'admission plus ou moins implicite que l'histoire saura en principe trancher entre le vrai et le faux, entre le réel et l'irréel, entre le réalisable et l'utopique. Autrement dit, le moderne attend en principe de l'histoire l'affirmation du réel, non sa contradiction. Ainsi avons-nous une séquence de contradictions, ou de noncontradictions, s'accompagnant d'une séquence de conceptions de la vérité, selon les ordres des ensembles de référence.

L'objectivité-1 semble être la première forme de l'objectivité qu'a connue la pensée occidentale. La réalité est alors conçue selon un mode de constitution qui suppose des variations d'instances subjectives dans un ensemble d'ordre un, soit individuel. Les principes matériels explicatifs des Ioniens, ou encore de l'Être parménidien, pourraient répondre à cette caractérisation. On notera par exemple que le choix de ces principes, ou la vérité de cet Être, ne sont pas à discuter, qu'on ne répond pas à des objections, mais qu'il y a plutôt une révélation issue d'un recueillement personnel. Il n'empêche qu'il s'y présente une forme de rationalisation nouvelle, selon laquelle le sujet est penseur, ou créateur de son monde de pensée, et que des démonstrations explicatives sont effectuées.

13. Dans cet article, la «modernité» s'identifie approximativement aux $\mathrm{XIX}^{\mathrm{e}}$ et $\mathrm{XX}^{\mathrm{e}}$ siècles. KaNT et HeGEL en marquent le seuil. 
Le caractère révolutionnaire du passage à l'ordre suivant (soit de l'ordre intrasubjectif à l'ordre intersubjectif pour la base de référence implicite) est manifeste chez Platon. Dans la République, Socrate s'entretient d'abord avec Polémarque et ils tentent ensemble de définir la justice. Ils réfléchissent en dialoguant, tout en constituant ensemble un espace, un lieu de pensée commun. Polémarque suggère que la justice consiste à faire du bien à ses amis et du mal à ses ennemis. Socrate passe alors à un degré réflexif supplémentaire. Supposons, dit-il, qu'un homme quelconque considère comme son ami quelqu'un déjà désigné par toi-même comme ennemi : alors, s'il suivait ton principe, il ferait du bien à ton ennemi et, de même, il ferait du mal à tes amis, et pourtant on devrait, conformément à ta définition, considérer cela comme juste. Bien sûr, il est admis implicitement par Socrate que la définition de Polémarque doit aussi valoir pour tout autre sujet qu'il pourrait désigner, tout comme d'ailleurs Polémarque, dialoguant avec Socrate, s'attend à ce que ce qu'il dit de vrai soit vrai aussi pour Socrate. Par exemple, la justice universelle comme idée doit prévaloir au niveau de Socrate et de Polémarque et au niveau de tous les sujets qu'ils peuvent désigner.

Kant, à la même question qui consiste à juger la définition proposée par Polémarque, aurait répondu qu'il suffit d'appliquer dans ce cas l'impératif catégorique, lequel se trouverait être l'explicitation formelle du raisonnement que Platon fait effectuer à Socrate ci-dessus. Platon aurait vraisemblablement estimé une telle explication redondante et il aurait jugé que l'universalité impliquée dans l'impératif kantien n'est qu'un autre nom pour la vérité. Pour Kant, au contraire, cette loi formelle a une signification particulière parce qu'il distingue essentiellement vérité pratique du niveau moral et vérité du savoir spéculatif de façon que cette dernière dépende explicitement de la première. Chez Platon cette distinction n'est pas conçue et la vérité lui apparaît une.

\section{Conceptions modernes de l'objectivité}

Les modernes identifient souvent l'objectivité à une objectivité fondée sur l'intersubjectivité. L'ordre d'objectivité explicité est donc généralement le deuxième, mais il peut l'être de différentes façons. Par exemple, Bachelard se trouve à décrire la double 
dimension de la base de référence quand il souligne que, sans le mathématicien, l'expérimentateur peut être victime de vues personnelles et que, sans l'expérimentateur, le mathématicien s'enferme dans l'abstraction ${ }^{14}$. Autrement dit, les actes expérimentaux du savant passent à l'objectivité quand il sont projetés dans un espace-temps abstrait constitué mathématiquement qui est le même pour tous les savants. Popper écrit pour sa part que non seulement l'objectivité de l'expérience scientifique est de l'ordre de l'intersubjectivité mais qu'en plus les énoncés protocolaires (qui décrivent les données dans un protocole d'observations convenu) sont révocables à tout moment parce qu'ils requièrent en principe le consensus des scientifiques ${ }^{15}$. Dans les faits, des arguments mathématiques peuvent être déterminants, ce qui rejoint alors le propos de Bachelard. On s'en tient donc à l'ordre référentiel intersubjectif quand il s'agit de définir explicitement l'objectivité valable dans les sciences expérimentales.

On peut cependant passer à l'ordre suivant quand on envisage la démarcation entre les sciences expérimentales et les sciences dites humaines. Le sociologue L. Goldmann par exemple la décrit comme suit. On parle de pensée objective dans les sciences physico-chimiques dans la mesure où les buts de ce savoir, soit la maîtrise de la nature par l'homme et la structuration catégoriale qui en résulte, sont les mêmes pour tous les groupes sociaux actuellement existants. Or la situation est radicalement différente pour les sciences humaines, qui ne peuvent faire abstraction du fait qu'il y a des différences entre les groupes sociaux ${ }^{16}$. Il y a, dans la caractérisation de cette démarcation, l'expression d'un changement d'ordre pour la base de référence dans la mesure où la variation référentielle sur les groupes sociaux apparaît déterminante pour la vérité objective, et non plus seulement sur un ensemble d'individus sans égard aux groupes distincts ${ }^{17}$. Ce nouvel ordre se manifeste

14. G. BACHELARD, Le rationalisme appliqué, op. cit., p. 3.

15. K.R. POPPER, La logique de la découverte scientifique, op. cit., Chapitre 1 , section 8.

16. L. Goldmann, Épistémologie et philosophie politique, Paris, Denoël-Gonthier, 1978, p. 24 sq.

17. On notera ici que la distinction entre les deux types d'objectivité mentionnés ne tient pas au fait que les sciences expérimentales puissent ou non valoir en fait pour toutes les différentes sociétés, en opposition au cas des sciences humaines. La distinction réside au contraire en ce que différentes sociétés ne se réduisent pas simplement à une communauté unique d'individus dans le cas des sciences humaines, par opposition aux sciences expérimentales. 
dans diverses expressions ou formulations. L'objectivité au même ordre, qui paraît d'abord propre aux sciences humaines, sera par exemple celle où la vérité se présente issue d'un déchiffrement des signes que les sociétés humaines en viennent, dans leurs cultures respectives, à produire ou à exprimer. On doit alors interpréter selon des sens multiples certains documents et tenir compte de l'existence du symbolique comme ordre distinct du réel phénoménal. La dimension référentielle requise ici peut être vue comme « historique », « humaine », « culturelle » ou encore «symbolique». La pensée philosophique peut par ailleurs être considérée comme relevant de cet ordre d'objectivité dans la mesure où la dimension historique lui est essentielle.

Mais cette dimension historique importe aussi à ceux qui tiennent un discours sur les sciences expérimentales quand ils s'avisent de tenir compte des changements conceptuels profonds qui touchent le domaine scientifique et modifient conséquemment les groupes de référence. Un auteur moderne, conscient du caractère historique du développement du savoir, peut parfois poser des conditions qui le reflètent. Ainsi Popper, invoquant la « discussion rationnelle » comme la méthode générale de la pensée philosophique, définit l'échange valable comme étant celui où l'on "dépasse sa propre solution, plutôt que de la défendre ${ }^{18}$, ce qui n'est pas sans rappeler ce qu'écrivait J. S. Mill : « Nos convictions... ne reposent pas sur d'autre sauvegarde qu'une invitation permanente adressée à tous de les démontrer non fondées ${ }^{19}$. On dépasse par là l'ordre simplement intersubjectif pour imposer en plus l'exigence d'un développemerıt à travers le changement des solutions déjà admises généralement sur une base intersubjective et l'exigence d'une attention constante pour les possibilités que le temps apporte. Nous voyons dans ce cas qu'un même auteur, par exemple Popper, peut caractériser explicitement l'objectivité comme étant basée sur l'intersubjectivité tout en admettant en général autre chose en plus pour constituer la vérité, soit le dépassement des formes du savoir. Ainsi le moderne exprime-t-il clairement le rôle de la référence intersubjective tout en laissant voir l'importance de la référence historique.

18. K.R. POPPER, op. cit., p. 13

19. J.S. MiLl, On Liberty, Londres, J. W. Parker \& Son, 1859, p. 40. 


\section{Autres considérations sur les ordres d'objectivité}

S'il apparaît que des auteurs contemporains ont reconnu assez nettement la spécificité des ordres référentiels correspondant à l'objectivité sur base intersubjective et à la vérité requérant une base historique (la première s'associant aux sciences expérimentales et la seconde plus particulièrement aux sciences humaines), est-il aussi clair qu'il y a bien une spécificité de l'objectivité-1, qui est plutôt relative à une base intrasubjective ? On pourrait avancer par exemple que rien n'empêcherait en principe un individu seul de développer une science expérimentale. Or il appert que si l'objectivité-1, qui admet une base de référence individuelle, suffit éventuellement quand il s'agit d'une démonstration mathématique ou purement logique, une base intersubjective, et donc l'objectivité-2, est en revanche essentielle pour les sciences dites de la nature, ou sciences expérimentales. Jean Piaget, par exemple, reconnaît la différence essentielle entre l'expérimentation et la déduction. Les conditions d'apprentissage humain pour la première se développent, selon lui, en retard par rapport à celles pour la seconde. Il considère la déduction comme une construction spontanée de l'intelligence, ce qui ne serait pas le cas pour l'expérimentation, qui exige une soumission à des instances extérieures ${ }^{20}$. Il constate chez l'enfant que les opérations déductives se constituent bien avant les conduites expérimentales et que ces dernières sont nettement subordonnées aux formes supérieures de celles-là. Cela peut signifier pour nous une confirmation indirecte, valant plutôt sur le plan épigénétique, de la généalogie historique exposée précédemment. En tout cas, cela donne des indications supplémentaires concernant la signification épistémologique des deux ordres d'objectivité.

D'autres aspects de l'objectivité-1 peuvent être trouvés dans la notion de «fait» selon Ernst Mach, à propos de laquelle il souligne la force immédiate de persuasion des sensations dans un moi et la prégnance des corps et des choses indépendants du moi, celui-ci étant un fait parmi des faits ${ }^{21}$. Dans ce cas, les corps extérieurs au moi, et le moi lui-même qui est désigné ici, sont des

20. J. PIAGET, Épistémologie des sciences humaines, Paris, Gallimard, 1970, pages 43-44.

21. E. MACH, La connaissance et l'erreur, trad. M. Dufour, Paris, 1908, p. 21 sq. 
faits dans un univers commun pour la constitution (ou la reconnaissance) desquels les sensations d'un sujet individuel suffisent.

Cela est à mettre en rapport avec le type d'objectivité requis par les mathématiques ou la logique. Rappelons la remarque de B. Russell selon laquelle un langage logiquement parfait serait un langage privé, impropre à la communication. Ce propos peut indiquer que la logique comme telle correspond à un ordre d'objectivité qui diffère de l'ordre intersubjectif et qui, plus précisément, devrait s'élaborer et se comprendre selon une base de référence d'abord individuelle. Ce langage serait impropre à la communication en autant qu'il s'agirait de communiquer autre chose que des résultats purement formels (de mathématique ou de logique). Tout ce dont la base d'objectivité est d'ordre supérieur à un ne pourrait d'abord s'y exprimer parce que cela équivaudrait à une réduction formelle complète, qu'ainsi une réalité expérimentale pourrait être réduite à du logique, et que même le sujet de l'expérience deviendrait un être purement logique. Le rapport peut nous apparaître profond entre le sens de cette remarque de Russell et la conception de Piaget selon laquelle la logique et les mathématiques résultent de la prise de conscience des conditions de l'action sur un objet quelconque et des coordinations possibles entre ces actions, que l'«objet quelconque » n'est dans l'ensemble de ces sciences qu'un invariant permanent, et les actions du sujet et leurs coordinations l'élément invariable qui détermine leur contenu ${ }^{22}$. Dans les deux cas, nous pouvons conclure que la base de référence individuelle est suffisante pour constituer l'ordre de réalité qui correspond à la logique et aux mathématiques, mais qu'elle ne suffit pas pour constituer l'ordre de réalité propre aux sciences expérimentales.

Les « faits » peuvent toutefois recevoir une autre interprétation qui leur donne un statut de réalités objectives-2, soit selon une base de référence intersubjective. Pour $\mathrm{J}$. Habermas par exemple le sens des "faits» ne peut pas être explicité sans référence aux discours ${ }^{23}$. Le fait peut alors acquérir une dimension spéculative, à

22. Certe conception s'oppose d'ailleurs à celle de F. Gonseth selon laquelle la logique est une "physique de l'objet quelconque ». Piaget insiste au contraire sur la démarcation entre le domaine logique et le domaine expérimental.

23. J. Habermas, Connaissance et intérêt, trad. G. Clémençon et J.-M. Brohm, Paris, Gallimard, 1976, p. 347. 
telle enseigne que certains épistémologues prétendent que le caractère de la distinction entre faits et théories est artificiel ${ }^{24}$. Il y a là, à mon avis, une confusion. Il continue d'y a voir une distinction essentielle à faire entre une théorie et un fait qui peut affecter cette théorie. Le fait, en tant que constatable intersubjectivement, ajoute à l'ordre d'objectivité que la théorie seule n'aurait pas. Une théorie par elle-même, épurée de tout fait, n'aurait de réalité que celle dont l'ordre d'objectivité équivaudrait à celui d'une logique ou d'une structure mathématique. T. S. Kuhn, incidemment, n'a pas tort de penser que le fait n'est pas une réalité absolue, indépendante du contexte social ou historique. Il faudrait toutefois distinguer essentiellement le fait dont le constat objectif infirme une théorie, ou encore lui donne naissance en créant les conditions de sa validité possible, et le fait tel que décrit, ou prévu à l'intérieur d'une théorie particulière, constituée dans la science expérimentale. La première espèce de fait peut posséder une objectivité d'ordre historique et la seconde ne peut excéder l'ordre d'objectivité de la théorie elle-même.

\section{Application au problème de l'induction}

Le problème épistémologique de l'induction dans les sciences expérimentales consiste à se demander si les lois scientifiques obtenues par induction à partir d'observations ou d'expérimentations particulières sont en principe justifiables et à quelles conditions elles peuvent l'être. C'est le passage du fait au droit, de l'expérience particulière à la pensée scientifique universelle, qui est en cause. Ce principe ne peut pas être justifié comme tel par l'expérience de fait puisque son objet est de tirer une législation universelle à partir de l'expérience ${ }^{25}$.

Comme le problème de l'induction en est un de justification et qu'il traduit la recherche d'un fondement, il peut recevoir ici une interprétation qui, je le pense, apportera une clarification importante.

24. Comme T.S. KuHN dans La structure des révolutions scientifiques (trad. L. Meyer, Paris, Flammarion, 1983, p. 83).

25. On a tenté de traiter l'induction des sciences expérimentales comme un cas d'application de la théorie des probabilités (notamment dans le positivisme logique). Le même problème de passage du fait empirique à la scientificité se pose dans ce cas encore. Voir à ce propos la critique de $\mathrm{K}$. R. POPPER dans La logique de la découverte scientifique, op. cit., pages 25-26. 
De même que la notion d'objectivité impliquée dans les sciences expérimentales a été mise en rapport, dans cet article, avec d'autres ordres d'objectivité dans une séquence particulière, il est possible d'établir une séquence où l'induction se trouve en rapport avec autre chose. Considérons la séquence suivante.

1. La déduction dans les mathématiques et la logique.

2. L'induction dans les sciences expérimentales.

3. La dialectique dans les savoirs de type philosophique.

Les différences essentielles entre les notions désignées par ces termes sautent d'abord aux yeux plutôt que le motif de leur continuité profonde. Le problème de l'induction des sciences expérimentales paraît justement tenir au fait qu'on ne peut traiter cette induction comme une déduction mathématique et que d'autre part on serait peu satisfait de comprendre l'induction selon le même registre qu'un passage dialectique, que les scientifiques préfèrent laisser aux philosophes ou aux métaphysiciens. Mon but n'est d'ailleurs pas de niveler les réalités diverses en les ramenant abusivement à un dénominateur commun. J'espère au contraire par le moyen de cette séquence souligner les différences essentielles, en les articulant néanmoins de façon à les rendre plus intelligibles. Il est ainsi possible de voir qu'à son niveau propre l'induction des sciences expérimentales reproduit le motif même que présente la déduction au niveau logico-mathématique, pourvu que l'on tienne compte correctement des différences d'ordres dans l'objectivité associable à ces différents savoirs.

La déduction mathématique suppose, à chaque étape d'une démonstration, une rétention des étapes précédentes en un sens de reconstructibilité pour le sujet mathématicien lui-même qui l'effectue. Il ne s'agit pas ici d'un credo de type intuitionniste, mais plutôt de la constatation générale que la saisie d'une étape dans une déduction mathématique implique la possibilité en principe de reconstituer la démarche raisonnée qui constitue la démonstration en ses différents moments. Comparons maintenant avec l'induction dans les sciences expérimentales. Celle-ci suppose une répétabilité de principe de l'expérience et du résultat expérimental. Ce que la rétention fait pour les moments du raisonnement individuel, la répétabilité le fait pour les sujets expérimentateurs. L'induction expérimentale traduit donc dans l'ordre intersubjectif ce que la déduction fait dans l'ordre intrasubjectif. 
Et il en va de même, mutatis mutandis, pour ce que les penseurs modernes ont appelé «dialectique», si l'on entend ce mot dans un sens général d'articulation entre les moments historiques successifs. En raison de la différence d'ordre, ce que suppose la notion de dialectique ne peut être décrit en termes de rétention intrasubjective ni de répétabilité intersubjective. Il le serait plutôt en opposition avec ce type de notion parce que le passage dialectique se décrit comme une rupture et un dépassement. Or il s'agit d'une rupture avec quelque chose établi selon un ordre intersubjectif d'objectivité parce qu'on se trouve à substituer à une réalité intersubjective à un moment de l'histoire, dans une communauté donnée, une autre réalité intersubjective correspondant à un autre moment de l'histoire, ou à une autre communauté distincte.

L'induction scientifique ainsi entourée de part et d'autre peut acquérir une signification essentielle très éclairante. D'une part, si nous comparons l'induction scientifique à la déduction mathématique ou logique, son exigence de répétabilité semble arbitraire et non fondée au regard des conditions requises de la déduction. Le type d'objectivité requis par le mathématicien impose un motif de continuité à un niveau plus élémentaire qui est celui de la référence individuelle. En comparaison, la vérité inductive d'une loi expérimentale semble reposer sur une sorte d'acte de foi du moment que sa confirmation demande un passage à l'autre sujet, dans une communauté intersubjective. La rigueur de l'induction expérimentale n'est cependant pas en soi inférieure à celle de la déduction, mais elle s'exerce pour des instances d'un ordre plus élevé. En ce sens la loi scientifique est aussi certaine pour la communauté scientifique que le théorème de mathématique peut l'être pour l'individu mathématicien. Celui-ci se sentira parfois fondé à critiquer le manque de rigueur du physicien éventuellement, mais il sera obligé de reconnaître la validité d'ensemble de la science physique.

Ce rapport du mathématicien au physicien se retrouve entre les physiciens et les philosophes, pourvu que la transposition soit faite complètement, c'est-à-dire en tenant compte de toutes les implications du changement d'ordre de la référence. La logique dialectique peut sembler très peu fiable aux yeux des physiciens parce qu'ils tentent de l'apprécier à la lumière des rapports intersubjectifs fondant l'objectivité, ou d'une répétabilité immédiate, alors qu'elle est d'ordre historique. Ainsi les tenants des sciences 
expérimentales à la recherche d'une explication peuvent s'estimer insatisfaits de la compréhension et des interprétations en usage dans les sciences historico-herméneutiques ou dans la philosophie. La communauté scientifique peut aussi juger et déprécier l'activité de compréhension de la communauté philosophique. En revanche, elle se sentira obligée de reconnaître une validité historique en quelque sorte à la philosophie en tant que forme de pensée rationnelle. La philosophie a par exemple pu contribuer au développement vers la pensée scientifique expérimentale ${ }^{26}$.

Le caractère problématique de l'induction dans les sciences expérimentales serait donc lié à une certaine confusion des ordres de savoirs. On pense d'abord à une sorte de validité logique formelle exigible au niveau des sciences expérimentales et, ne la trouvant pas telle quelle, on érige en problème l'absence de certification en soi qui pourtant se retrouve à tous les ordres de savoirs rationnels. La validité absolue n'est exigible nulle part.

\section{Conclusion : d'autres possibles}

D'après le Vocabulaire de Lalande (sous «Possible»), l'impossible se dit de ce qu'on sait objectivement ne pouvoir être. Puis de là découle une appréciation de ce qui reste possible.

Si l'objectivité est relative et s'il y a plusieurs sortes d'objectivités, précisément plusieurs ordres de l'objectivité, nous n'avons pas à nous en tenir à l'énoncé d'un possible objectif particulier. Ainsi, pour prendre un exemple pittoresque, atteindre la galaxie d'Andromède pour un vaisseau habité est physiquement impossible. Cette impossibilité est relative à l'objectivité- 2 de la science actuelle. On la relativise aussitôt en passant à une objectivité-3, qui fait plutôt dire : il est impossible dans l'état actuel de nos connaissances et de nos moyens techniques d'aller en personne dans une autre galaxie que la nôtre. A fortiori, si nous passons à une objectivité d'ordre supérieur, une appréciation d'impossibilité pourra être dépassée.

26. On reconnaît par exemple le rôle de la philosophie cartésienne dans le développement des conceprs de la physique et celui de l'aristotélisme dans le développement de la pensée biologique. 
Bien sûr, s'il ne s'agissait que de la non-contradiction, tout deviendrait possible à la seule condition de déniveler pour éviter le heurt contradictoire. Toutefois les possibilités qui se dégagent d'une généralisation de la notion d'objectivité n'équivalent pas à toute admission non critique. J'espère avoir démontré dans cet article ${ }^{27}$ que c'est en quelque sorte par l'objectivité d'ordre supérieur qu'on peut reconnaître l'existence des ordres d'objectivité puisque ces ordres représentent l'invariance de quelque chose de plus essentiel que ce qui apparaît dans l'objectivité au sens courant. Limiter absolument le possible par une objectivité d'un ordre donné est sûrement abusif. On ne peut davantage, par exemple, laisser déterminer les possibilités du $\mathrm{XX}^{\mathrm{e}}$ siècle à partir d'un point de vue du XVII ${ }^{e}$ siècle. Il sera aussi difficile à un moderne d'admettre l'ordre qui dépasse la modernité qu'il l'aurait été à un classique d'admettre l'ordre moderne.

Département de physique, Collège Montmorency

\section{BIBLIOGRAPHIE}

BACHELARD, G., Le rationalisme appliqué, Paris, P. U. F. 1949.

GOLDMANN, L., Épistémologie et philosopbie politique, Paris, Denoël-Gonthier, 1978.

HABERMAS, J., Connaissance et intérêt, trad. G. Clémençon et J.-M. Brohm, Paris, Gallimard, 1976.

KANT, E., Critique de la raison pure, trad. J. Barni, Paris, Flammarion, 1976.

KUHN, T.S., La structure des révolutions scientifiques, trad. L. Meyer, Paris, Flammarion, 1983.

LALANDE, A., Vocabulaire technique et critique de la philosopbie, Paris, P. U. F., $14^{e}$ édition, 1983.

MACH, E., La connaissance et l'erreur, trad. M. Dufour, Paris, 1908.

MILL, J.S., On Liberty, Londres, J. W. Parker \& Son, 1859.

27. Je suis conscient que les notions présentées dans cet article, dans la mesure même de leur originalité, n'ont pu être suffisamment explicitées ou élucidées. Je me permets cependant d'ajouter que d'autres publications viendront, dans un proche avenir, clarifier ces notions davantage et montrer leur fécondité. 
PIAGET, J., Épistémologie des sciences humaines, Paris, Gallimard, 1970.

PLATON, La République, trad. R. Baccou, Paris, Flammarion, 1966.

POPPER, K.R., La logique de la découverte scientifique, trad. N. ThyssenRutten et P. Devaux, Paris, Payot, 1973.

RUSSELL, B., Méthode scientifique en philosopbie, trad. P. Devaux, Paris, Vrin, 1929.

Signification et Vérité, trad. P. Devaux, Paris, Flammarion, 1959. 